

\title{
¿EI desarrollo del mercado accionario genera crecimiento económico en México? Un análisis de series de tiempo*
}

\author{
Francisco López Herrera** y Domingo Rodríguez Benavides***
}

\section{RESUMEN}

En este trabajo se presentan los resultados del análisis de cointegración efectuado para averiguar si existe una relación estable y de equilibrio de largo plazo entre el índice de precios y cotizaciones (IPC) de la Bolsa Mexicana de Valores y el índice de volumen de la producción industrial (IVPI). Se argumenta en la bibliografía que las expectativas formadas en el mercado de valores tienden a incidir en los valores futuros de los indicadores de la actividad económica, por ejemplo, véase a Ron (2001). A partir de la estimación de un modelo de corrección del error, se encuentra evidencia que apoya la hipótesis de una relación estable y de largo plazo entre ambas variables, en el mismo sentido que los hallazgos de Ron (2001) para México. Sin embargo, encontramos únicamente evidencia de una causalidad débil que va del IPC al IVPI para un periodo más reciente que el abordado por dicho autor.

Palabras clave: mercado accionario, actividad económica, desarrollo financiero, crecimiento económico, cointegración.

Clasificación JEL: D53, N26, O16.

\begin{abstract}
This paper shows the results of cointegration analysis carried out to look for a stable and steady long-run relationship between the Mexican Stock Market Index (IPC) and the Volume Index of Industrial Production (IVPI). In the literature is argued that the expectations formed in the stock market tend to affect the future values of the indicators of economic activity, for example see Ron (2001). By means of an error correction model we show evidence supporting the hypothesis of a stable, long-run relationship between the Stock Market and Industrial production Indexes; a result consistent with the findings of Ron (2001). Nevertheless, we find out only weak evidence about causality running from IPC to IVPI for a more recent period than that covered by Ron's study
\end{abstract}

Key words: stock market, economic activity, financial development, economic growth, cointegration.

Classification JEL: D53, N26, O16.

\footnotetext{
* Agradecemos a dos dictaminadores anónimos sus comentarios y sugerencias con el fin de mejorar nuestro trabajo. Cualquier error u omisión que exista es responsabilidad exclusiva de los autores. ** División de Investigación FCA-UNAM. Doctor en economía por la Facultad de Economía, UNAM: francisco_lopez_herrera@yahoo.com.mx.*** Profesor de la UAM-Azcapozalco. Candidato a doctor en economía por la Facultad de Economía, UNAM: domr@economia.unam.mx.
} 


\section{INTRODUCCIÓN}

La discusión académica sobre el vínculo entre el desarrollo financiero y el crecimiento económico se remonta a principios del siglo XX con los trabajos de Schumpeter (1911). La relación entre estas variables ha generado gran interés y un fuerte debate entre los economistas a partir de aquel entonces. De acuerdo con Guha y Mukherjee (2008), el debate ha girado básicamente en torno de dos cuestiones: $i$ ) se ha discutido si hay una relación entre desarrollo financiero y crecimiento económico, y ii) cuál es la naturaleza y dirección de la relación causal entre ambas variables: ¿el desarrollo del sector financiero promueve el crecimiento económico, o el desarrollo financiero es resultado del crecimiento económico?

En términos generales, la bibliografía sobre el tema se puede clasificar en tres grandes visiones relativas a la importancia del sector financiero en el crecimiento económico. La primera considera el sector financiero como un elemento fundamental para el crecimiento; como exponentes destacados de esta corriente pueden citarse: Schumpeter (1911), Goldsmith (1969), McKinnon (1973), Shaw (1973), Odedokun (1996), King y Levine (1993a y 1993b). También hay quienes adoptan una posición diametralmente opuesta, pues ven a dicho sector como un factor sin mucha importancia relativa para el crecimiento, por ejemplo, Robinson (1952), Lucas (1988) y Stern (1989). Por último, la tercera visión se centra en el impacto potencialmente negativo del funcionamiento del sector financiero sobre el crecimiento, entre otros, Van Wijnbergen (1983) y Buffie (1984). En forma paralela a esas visiones se ha llevado a cabo una cantidad considerable de estudios empíricos, sin embargo, no ha sido posible establecer una posición definitiva pues en algunos de aquellos se ha encontrado evidencia mixta respecto a los efectos del desarrollo financiero sobre el crecimiento económico: en algunos casos no se observa una relación positiva, como se esperaría de acuerdo con la hipótesis McKinon-Shaw, y en otros análisis ni siquiera se detecta una relación significativa, como lo destaca Xu (2000).

No obstante el avance mostrado por los desarrollos teóricos en esta área, éstos tampoco establecen de forma clara la dirección de la causalidad entre desarrollo financiero y crecimiento económico. Tal es el caso de McKinnon (1988), quien se cuestiona: “¿Cuál es la causa y cuál es el efecto? ¿Es el sector financiero un promotor del desarrollo económico, o simplemente sigue al crecimiento del producto real que es generado por otros factores?". Hasta la fecha no existe consenso de que el desarrollo financiero determine el crecimiento económico, si esto 
se da a la inversa, o bien si ambos procesos se determinan simultáneamente. Como consecuencia de lo anterior, se puede encontrar en la época más reciente una serie de trabajos empíricos que han intentado establecer la dirección de la causalidad entre desarrollo financiero y crecimiento económico. Algunos de estos trabajos tienden a confirmar una asociación positiva entre el desarrollo financiero y el crecimiento económico.

Dentro de la discusión acerca de que si el desarrollo financiero propicia crecimiento económico o a la inversa, se puede enmarcar la creciente importancia de los mercados de capitales en los países desarrollados alrededor del mundo en las décadas pasadas, la cual ha redirigido el interés de los investigadores para explorar las relaciones entre los mercados de capitales de esos países y el crecimiento económico, como lo destacan Guha y Mukherjee (2008). A pesar del gran interés suscitado en torno de las relaciones entre el desarrollo de los sistemas financieros y el crecimiento de la economía, algunas cuestiones no están del todo claras en el análisis teórico y empírico de este importante vinculo; por ejemplo, destaca el hecho de que no se sabe con precisión si los mercados bursátiles liberalizados y catalogados como emergentes han resultado realmente importantes o no para el crecimiento económico.

El análisis entre estas dos variables es significativo desde distintos ángulos, por ejemplo, se tiende a dar por hecho que el desarrollo del mercado de valores propicia un mayor nivel de actividad económica, al suponer que provee mayor liquidez a los empresarios, por lo cual éstos pueden emprender nuevos proyectos de expansión y crecimiento, alentando de esta manera la actividad económica.

De acuerdo con Mishkin (2001) y Caporale et al. (2004), los mercados de capitales organizados y administrados estimulan las oportunidades de inversión a partir del financiamiento de proyectos productivos que alientan la actividad económica, movilizan el ahorro doméstico, asignan de manera más eficiente el capital, ayudan a diversificar el riesgo y a facilitar el intercambio de bienes y servicios. Indudablemente, se espera que los mercados de capitales incrementen el crecimiento económico no sólo a partir del incremento de la liquidez de los activos financieros, como se ha planteado, sino también de la diversificación del riesgo doméstico y global, y de la promoción de mejores decisiones de inversión, como lo plantea Osei (2005). Bajo esa perspectiva, se puede considerar entonces que los mercados de capitales son un buen indicador para pronosticar la actividad económica futura, y contribuyen a describir la incidencia de los precios actuales de los activos en la actividad económica futura. En la dirección contraria, 
la relación entre el desarrollo del mercado de valores y el crecimiento económico real se puede explicar como la forma en la que algún cambio en el comportamiento del mercado de capitales eventualmente cambiará el costo del capital. Si el costo de capital de la empresa se incrementa aumentando el costo de la inversión, se puede esperar que esto desacelere el crecimiento de la economía ya que disminuye la rentabilidad de las inversiones en activos reales y las empresas pueden no llevar a cabo o simplemente cancelar proyectos de inversión cuya rentabilidad no les es atractiva. En el caso de una reducción del costo de capital cabría esperar la situación contraria.

Por tanto, los mercados de capitales desempeñan un importante papel en la asignación del capital para el sector corporativo y, a su vez, estimulan la actividad económica real. Muchos países enfrentaron restricciones financieras, particularmente los países en desarrollo, cuando los préstamos bancarios se restringieron a favorecer a ciertos grupos de inversionistas y compañías. Esta limitación puede también reflejar restricciones en el mercado de crédito, como lo señalan Mirakhor y Villanueva (1990).

De igual forma, los mercados eficientes de capital proveen lineamientos como un medio para mantener una política monetaria apropiada por medio de la emisión y recompra de valores gubernamentales en el mercado de dinero, lo cual constituye un importante paso hacia la liberalización financiera. De manera similar, los mercados de capital bien organizados pueden modificar el patrón de demanda de dinero, y coadyuvar a la creación de liquidez que finalmente aliente el crecimiento económico, según sostienen Caporale et al. (2004).

De acuerdo con Levine (1997) y Bencivenga y Smith (1991), mercados más líquidos pueden crear inversiones de largo plazo y, por tanto, crecimiento económico mediante un costo de transacción menor. En el mismo sentido, Levine y Zervos (1998) subrayaron que la liquidez de los mercados de capitales predice positivamente el crecimiento económico agregado. Rajan y Zingales (1998) argumentan que el tamaño del mercado de capitales está correlacionado con el crecimiento de las empresas financieras.

Tradicionalmente, los teóricos del crecimiento han creído que no existe correlación entre el mercado de capitales y el crecimiento económico. En esta línea, Singh (1997) sostuvo que los mercados de valores no son instituciones necesarias para alcanzar altos niveles de desarrollo económico. Otros han visto al mercado de capitales como un peligroso agente para el desarrollo económico debido a su susceptibilidad a las fallas del mercado, lo cual a menudo se manifiesta en los mercados en desarrollo, según Singh (1997). 
Contrariamente a la visión tradicional, existe evidencia que brinda apoyo a la hipótesis de que existe correlación de largo plazo entre el desarrollo del mercado de capitales y el crecimiento económico, como es el caso que muestran Shahbaz, Ahmed y Ali (2008). Aunque la bibliografía para probar esta hipótesis ha venido en aumento, inclusive en los países en desarrollo, Pardy (1992), en su trabajo seminal, ha argumentado que los mercados de capital en los países menos desarrollados son capaces de movilizar el ahorro doméstico y asignar los recursos más eficientemente.

Dentro de dicho contexto, en este trabajo abordamos únicamente la cuestión de la causalidad entre el desarrollo del mercado de capitales y el crecimiento económico usando datos de series de tiempo para México. El objetivo de nuestro trabajo es determinar en primera instancia si existe en México una relación de largo plazo entre el crecimiento económico y el desarrollo del mercado de capitales; asimismo buscamos evidencia econométrica sobre la posible dirección de la causalidad entre ambas variables. Por ejemplo, si ambas variables se determinan simultáneamente podrían generar una suerte de círculo virtuoso. De ahí, la necesidad de establecer si en el caso de México hay una dirección de causalidad entre ambas variables. De esta manera, el identificar la posible dirección de la causalidad entre las variables en cuestión puede contribuir al diseño e implantación de políticas económicas que promuevan el crecimiento económico.

Con tal objetivo, empleamos las técnicas del análisis de cointegración y de la causalidad en el sentido de Granger para estudiar la relación entre el índice de precios y cotizaciones de la Bolsa Mexicana de Valores y el índice de volumen de la producción industrial, donde consideramos a estas variables como indicadores indirectos (proxies) del desarrollo del mercado de valores y del crecimiento económico de México, respectivamente. ${ }^{1}$

El artículo está estructurado de la siguiente forma. En la sección I se ofrece una breve revisión de una muestra relevante de trabajos empíricos sobre el tema. Posteriormente, la sección II describe los datos y la metodología econométrica empleada para sustentar el análisis empírico. La sección III presenta la evidencia empírica y por último se presentan las conclusiones de nuestra investigación.

\footnotetext{
${ }^{1}$ Es posible recurrir a otros indicadores del desarrollo del mercado de valores, como son tamaño, medido por la razón valor del mercado a PIB, de liquidez, ya sea por la razón monto operado en el mercado de valores a PIB, o bien a partir de la construcción de algún índice con estas variables; por el momento, nosotros sólo nos limitamos a averiguar la relación entre IPC e IVPI pues captan de manera sintetizada los efectos de nuestro interés.
} 


\section{REVISIÓN DE LA BIBLIOGRAFÍA}

En diferentes trabajos se ha postulado que la actividad en el mercado de valores y la actividad económica real guardan una relación de equilibrio y que el primero puede ser un buen predictor del segundo. Entre los primeros trabajos que investigan este importante vínculo se encuentra el de Fama (1981), en el que se ofrece evidencia estadística de la existencia de una relación positiva entre los rendimientos del mercado de valores y la futura actividad económica real, gastos de capital, tasa de rendimiento del capital y producción. Fama concluyó que la tasa de crecimiento de la producción industrial era la única variable real que presenta una fuerte relación con los rendimientos del mercado de valores.

Además del trabajo de Fama (1981), se han llevado a cabo otros trabajos para estudiar el vínculo entre la actividad económica y el comportamiento del mercado accionario estadounidense. Valiéndose de un modelo vectorial de autorregresiones con promedios móviles (VARMA), James, Koreisha y Partch (1985) analizan las relaciones de causalidad entre los rendimientos accionarios, la actividad real, la oferta monetaria y la inflación, encontrando evidencia de que los rendimientos de las acciones estadounidenses pueden anticipar el comportamiento de la actividad real y de la base monetaria. Los resultados de Fama (1990), Schwert (1990) y Lee (1992) sugieren que los rendimientos del mercado accionario estadounidense pueden constituir un indicador que señala anticipadamente cambios en la actividad económica real, pues encuentran evidencia significativa de que dichos rendimientos tienen una relación positiva con las tasas futuras de crecimiento de la producción.

No obstante la evidencia encontrada en favor de la existencia de una relación significativa entre el comportamiento del mercado de valores estadounidense y la actividad económica de ese país, los resultados de la investigación de Binswanger (2000) muestran que las variaciones de los precios accionarios en Estados Unidos han dejado de anticipar el comportamiento de la actividad económica real desde el auge del mercado accionario que se dio a inicios de la década de los ochenta en ese país.

El trabajo de Thornton (1993) destaca la importancia de los precios accionarios en el producto real del Reino Unido. En otro trabajo, Choi et al. (1999) analizan la relación entre los rendimientos rezagados del mercado de valores y la tasa de crecimiento de la producción industrial para los países integrantes del G-7, utilizando las pruebas de cointegración y causalidad. El fundamento teórico de esta relación se basa en el modelo de valuación del flujo de efectivo desconta- 
do, que establece que el índice de precios del mercado accionario refleja las expectativas de los inversionistas acerca del comportamiento futuro de las variables económicas, tales como las ganancias corporativas o su variable proxy agregada, la producción industrial. Si en promedio estas expectativas son correctas, los rendimientos rezagados del mercado accionario deberían estar correlacionados con la tasa contemporánea de crecimiento de la producción industrial. En otras palabras, el rendimiento real del mercado accionario debería proveer información sobre la evolución de la producción industrial. Los resultados muestran una alta correlación entre la tasa de crecimiento de la producción industrial y los rendimientos rezagados del mercado de valores.

Aylward y Glen (2000) presentan los resultados de un estudio en el que analizan si el comportamiento de los precios accionarios constituye un indicador anticipado en 23 países (G-7, Australia y 15 países con mercados emergentes). Esos estudiosos presentan evidencia mostrando que para la mayoría de los países que estudiaron, los cambios en los precios de las acciones son significativamente un indicador anticipado del comportamiento del PIB, del consumo y de la inversión.

Habibullah et al. (2000) hacen uso de una prueba de causalidad de Granger propuesta por Toda y Yamamoto (1995) para probar la relación causal entre los rendimientos del mercado de valores y cinco variables macroeconómicas: oferta de divisas, ingreso nacional, nivel de precios del consumo, tasa de interés y una tasa de cambio real efectiva. El objetivo de la investigación busca determinar si los rendimientos del mercado de valores son un indicador líder de la actividad económica futura. Las conclusiones obtenidas por esos autores fueron: a) la oferta monetaria es un elemento que explica parte del comportamiento del nivel de precios del mercado de valores; $b$ ) el nivel de precios del mercado de valores afecta el ingreso nominal; $c$ ) el nivel de precios del mercado de valores afecta el nivel de precios del consumo; $d$ ) la tasa de interés afecta el nivel de precios del mercado de valores, y $e$ ) el nivel de precios del mercado de valores afecta el tipo de cambio real.

Por su parte, Hondroyannis y Papapetrou (2001) incluyen el comportamiento de la producción industrial en su estudio sobre las relaciones dinámicas entre el mercado accionario griego y otros indicadores de la actividad económica (tasa de interés, tipo de cambio, precios reales del petróleo y el comportamiento real del mercado accionario de Estados Unidos medido por el indicador S\&P 500), analizando las observaciones de esas variables en el periodo que va desde enero de 1984 hasta septiembre de 1999. Esos autores no detectan la existencia 
de una relación de largo plazo entre las variables estudiadas al efectuar las pruebas sugeridas por el método de Johansen (1988 y 1992) Johansen y Juselius (1990). Por lo anterior, deciden llevar a cabo el análisis de la dinámica de esas relaciones centrándose en el comportamiento de dichas variables en el corto plazo y basándose en el enfoque proporcionado por los modelos de vectores autorregresivos (VAR). Al aplicar el análisis de impulso respuesta, Hondroyannis y Papapetrou encuentran como uno de sus principales resultados que un incremento en los rendimientos reales del mercado accionario griego no conduce necesariamente a un nivel más elevado de la producción industrial.

En su análisis de la relación entre desarrollo financiero y crecimiento económico en la India para el periodo de 1996 a 2007, Guha y Mukherjee (2008) efectúan también la prueba de causalidad en el sentido de Granger propuesta por Toda y Yamamoto (1995). En sus resultados destaca el hallazgo de la existencia de bidireccionalidad en la relación entre la capitalización de la bolsa de valores de Bombay y la tasa de crecimiento del PIB, ambas variables medidas en términos reales.

Por su parte, Shahbaz, Ahmed y Ali (2008) llevan a cabo un estudio de esa misma relación, pero para el caso de Paquistán. Dichos estudiosos llevan a cabo una prueba de cointegración basándose en un modelo autorregresivo con rezagos distribuidos, utilizando para ello datos anuales de 1971 a 2006, y encuentran una fuerte relación con bidireccionalidad en el sentido de la causalidad entre ambas variables.

\section{DATOS Y METODOLOGÍA ECONOMÉTRICA}

Tomando en cuenta los resultados que han alcanzado diversos estudiosos de la relación existente entre el comportamiento del mercado de valores y la actividad económica "real", para la medición de esta última variable en el caso de México es posible utilizar el índice de volumen de la producción industrial (IVPI), en tanto que para estimar la actividad bursátil se puede hacer uso del índice de precios y cotizaciones (IPC) que publica la Bolsa Mexicana de Valores (BMV).

De acuerdo con el INEGI, el indicador mensual de la actividad industrial, medido a partir del IVPI, se expresa mediante un índice de cantidades de formulación Laspeyres, que tiene su base en el año de 1993, el cual refleja el volumen real de la producción obtenida en el mes. El marco conceptual y metodológico utilizado es semejante al empleado en el Sistema de Cuentas Nacionales de México (SCNM). Se calculan índices de volumen físico para 209 subgrupos de un 
total de 254 que integran las 57 ramas de actividad económica, incluidas en este indicador, agrupándolas primero en las nueve divisiones industriales, y después en cuatro grandes divisiones: minería, industria manufacturera, construcción y electricidad, gas y agua.

Con el fin de mostrar la evidencia empírica se utilizan dos series con una frecuencia mensual y un horizonte temporal, que comprende de diciembre de 1987 a febrero de 2008, para un total de 243 observaciones. La gráfica 1 muestra en logaritmos la evolución en el tiempo de ambas variables.

\section{Gráfica 1. Índice de volumen de la producción industrial (IVPI) e índice de precios y cotizaciones (IPC), 1987:12-2008:02}

(En logaritmos)

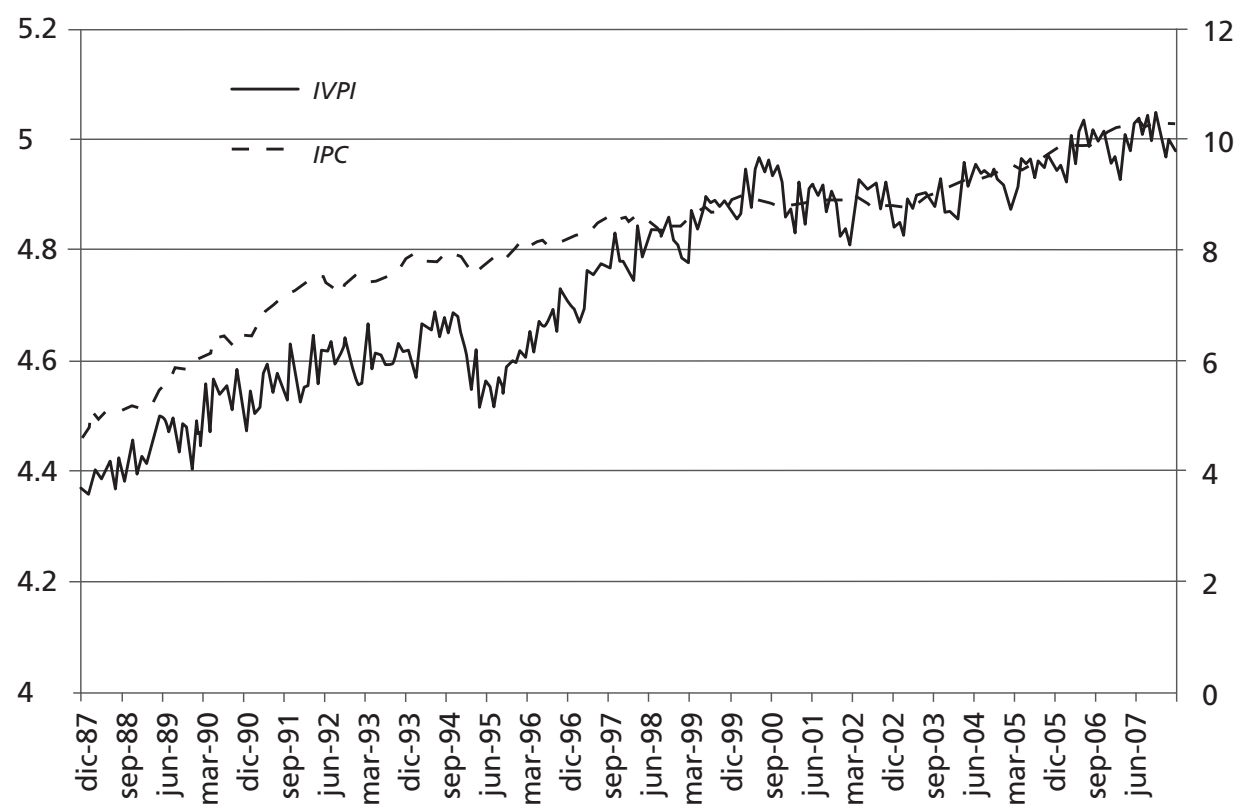

Como se puede apreciar, ambas variables tienden a moverse en forma conjunta, lo cual permite suponer la existencia de alguna relación de largo plazo en el tiempo.

El modelo econométrico que se pretende estimar es el siguiente

$$
i v p i_{t}=\beta_{0}+\beta_{1} i p c_{t}+u_{t}
$$


Donde ivpi es el logaritmo natural del índice de volumen de la producción industrial e ipc es el logaritmo natural del índice de precios y cotizaciones de la Bolsa Mexicana de Valores. Para la estimación del modelo econométrico (1) se emplearon series de tiempo mensuales tanto del índice de volumen de la producción industrial (IVPI), con información de Banco de México, como del índice de precios y cotizaciones (IPC) de la Bolsa Mexicana de Valores (BMV) del periodo antes mencionado.

Con el fin de identificar si las series incorporadas en (1) se comportan como paseos aleatorios, se llevaron a cabo pruebas de raíces unitarias. De acuerdo con la prueba Dickey-Fuller Aumentada (ADF) la hipótesis de una raíz unitaria se prueba a partir de la estimación del siguiente modelo:

$$
\Delta y_{t}=\alpha_{0}+\gamma y_{t-1}+\sum_{i=2}^{p} \beta_{i} \Delta y_{t-i+1}+\varepsilon_{t} .
$$

En la anterior especificación, el interés radica en probar la hipótesis nula: $\gamma=0$. Si $\gamma=0$, entonces se dice que la serie $y_{t}$ tiene una raíz unitaria.

Otra de las pruebas de raíz unitaria que empleamos es la prueba de Kwiatkowski, Phillips, Schmidt y Shin (KPSS), en la cual, por lo contrario, la hipótesis nula es que la serie de tiempo es estacionaria y, por ende, la hipótesis alternativa es que posee una raíz unitaria. Esta prueba toma en cuenta que la varianza de la suma parcial de los residuos de un modelo auxiliar como:

$$
z_{t}=\alpha+\delta t+\varepsilon_{t}
$$

debería ser baja en el caso de que la serie en cuestión sea estacionaria en tendencia y alta en el caso de que la serie sea estacionaria en diferencia (Chumacero, 2000). Teniendo en cuenta lo anterior, se construye un estadístico utilizando dicha suma parcial de los residuos y se comparan los valores críticos con los reportados en Kwiatkowsky et al. (1992).

Por otra parte, la técnica empleada para probar cointegración entre las series fue la sugerida por el método de Johansen, la cual prueba el rango de $\Gamma_{k}$ la matriz de parámetros asociada al vector de rezagos en los niveles de las variables. Esta técnica especifica el modelo de corrección del error (MCE) de un VAR de $m$-variables para un vector de series de tiempo $X_{t}$ como:

$$
\Delta X_{t}=\Gamma_{1} \Delta X_{t-1}+\Gamma_{2} \Delta X_{t-2} \ldots+\Gamma_{k-1} \Delta X_{t-k+1}+\Gamma_{k} X_{t-k}+\Phi D_{t}+v_{t}
$$


donde $\Gamma_{k}$ define la "solución en niveles" de largo plazo en la ecuación (4) (véase Cuthbertson et al. (1992), y $k$ es lo suficientemente grande para asegurar que $v_{t}$ sea un vector de ruido blanco gaussiano que se distribuye idéntica e independientemente con media cero y varianza finita.

De esta forma, la técnica prueba el rango de $\Gamma_{k}$, la matriz de parámetros asociada al vector de rezagos en los niveles de las variables. Mientras que $\Phi$ es una matriz cuadrada que estima el impacto de los elementos deterministas incorporados en el modelo dado en (4).

\section{EVIDENCIA EMPÍRICA}

El cuadro 1 muestra los resultados de las pruebas de raíces unitarias para las series en cuestión. Las series ivpi e ipc comprenden el periodo que va de diciembre de 1987 a febrero de 2008.

Como se puede apreciar en el cuadro 1, la prueba Dickey-Fuller Aumentada (ADF) no permite rechazar la hipótesis nula de la raíz unitaria para la serie del índice de volumen de la producción industrial (IVPI) al nivel de significación de $1 \%$, pero sí al 5\% de significancia. Mientras que los resultados de la prueba para la serie del índice de precios y cotizaciones $\left(i p c_{t}\right)$ tampoco permiten rechazar la hipótesis nula de raíz unitaria en los niveles de esta serie.

\section{Cuadro 1. Pruebas Dickey-Fuller aumentada para las series (1990:1-2004:4)}

\begin{tabular}{cccccc}
\hline Serie & $t_{a 1}$ & $t_{\gamma /}$ & Rezagos & $5 \%$ & $1 \%$ \\
\hline ivpi ${ }_{t}$ & $-3.669 *$ & 10.615 & 4 & -3.494 & -4.134 \\
$\Delta i v p i_{t}$ & $-5.902 * *$ & 7.160 & 4 & -1.947 & -2.608 \\
$i p c_{t}$ & -3.380 & 5.554 & 4 & -3.494 & -4.134 \\
$\Delta i p c_{t}$ & $-9.243 * *$ & -4.627 & 3 & -1.947 & -2.608 \\
\hline
\end{tabular}

${ }^{*} t_{a 1}$ representa el $t$-estadístico de la prueba Dickey-Fuller, $t_{\gamma /}$ el $t$-estadístico del último rezago. Pruebas realizadas en Eviews 5.1. 
Cuadro 2. Pruebas KPSS para las series ${ }^{a}$

\begin{tabular}{clcccccc}
\hline & \multicolumn{2}{c}{ Estadísticos } & \multicolumn{5}{c}{ Valores críticos } \\
Variable & $\eta_{\mu}$ & $\eta_{\tau}$ & \multicolumn{2}{c}{$\eta_{\mu}$} & & $\eta_{\tau}$ \\
& & & $5 \%$ & $1 \%$ & $5 \%$ & $1 \%$ \\
\hline$y_{t}$ & $1.8959 * *$ & $0.4630 * *$ & 0.463 & 0.739 & 0.146 & 0.119 \\
$\Delta y_{t}$ & 0.1155 & 0.0396 & 0.463 & 0.739 & 0.146 & 0.216 \\
$i p c_{t}$ & $1.7890 * *$ & $0.4630 * *$ & 0.463 & 0.739 & 0.146 & 0.119 \\
$\Delta i p c_{t}$ & $0.5086 *$ & $0.1610 *$ & 0.463 & 0.739 & 0.146 & 0.216 \\
\hline
\end{tabular}

a Prueba realizada con 12 rezagos. $\eta_{\mu}$ y $\eta_{\tau}$ representan los estadísticos de la prueba, donde la hipótesis nula considera que la serie es estacionaria en nivel o alrededor de una tendencia determinística, respectivamente. Los asteriscos * $y$ ** indican que la hipótesis nula se rechaza tanto al $1 \%$ como al $5 \%$ de significancia. Pruebas realizadas en J-Multi 4.23.

Por su parte, la prueba KPSS, en cualquier caso, ya sea asumiendo que la serie es estacionaria en nivel o alrededor de una tendencia, tiende a rechazar la hipótesis nula de estacionariedad en las series, lo cual tiende a confirmar los anteriores resultados de que ambas series poseen una raíz unitaria (cuadro 2).

Teniendo en cuenta que ambas variables poseen una raíz unitaria, procedimos a realizar la prueba de cointegración de Johansen entre ambas series. Los resultados de la prueba de Johansen se muestran en el cuadro 3.

Una vez que se verificó la existencia de cointegración entre las variables en cuestión, estimamos el modelo de corrección del error (MCE) con seis rezagos, en el cual se incorporaron tanto dummies estacionales centradas como tres dummies de pulso, para el mes de abril de 1995, y los meses de agosto y septiembre de 1998. Estos elementos deterministas generalmente tienden a incorporarse con fines de ajuste en el MCE, la significación de las dummies estacionales centradas dan cuenta del posible patrón estacional presente en las serie del índice de volumen de la producción industrial (IVPI), mientras que las dummies de pulso dan cuenta de la existencia de algunas rupturas en las series.

La incorporación de variables dummy en el procedimiento de Johansen (1988) modifica los valores críticos de las pruebas estadísticas (Mosconi, Johansen y Nilsen, 2000). Para la estimación de los nuevos valores críticos se utilizó el programa Cats in Rats, versión 2. En el cuadro 3 se presentan los resultados de la prueba de la traza con los nuevos valores críticos, confirmando la presencia de un vector cointegrante entre las variables en cuestión. 
Cuadro 3. Resultados de la prueba de la traza de Johansen ${ }^{a}$

\begin{tabular}{lcccccc}
\hline Periodo & $H_{0}$ :rango $=p$ & $-T \sum_{i=r+1}^{p} \ln \left(1-\hat{\lambda}_{r+1}\right)$ & Valor- $\mathrm{p}$ & $90 \%$ & $95 \%$ & $99 \%$ \\
\hline $\begin{array}{l}\text { 88:04- } \\
\text { 08:02 }\end{array}$ & $p=0$ & $31.77 * *$ & 0.0006 & 17.98 & 20.16 & 24.69 \\
& $p \leq 1$ & 5.66 & 0.2269 & 7.60 & 9.14 & 12.53 \\
\hline
\end{tabular}

a Los asteriscos * $y$ ** indican el rechazo de la hipótesis nula al 5 y al 1\% de significación, respectivamente.

* Indica los valores de los estadísticos corregidos y sus valores-p correspondientes para muestras pequeñas. Valores calculados con 20000 repeticiones para una muestra de 250 observaciones, incorporando dummies estacionales centradas y las dummies de pulso descritas en el texto.

El vector cointegrante estimado a partir de la técnica de Johansen se muestra en el cuadro 4:

Cuadro 4. Vector cointegrante estandarizado para $\beta_{0}+\beta_{1} i p c_{t}$

\begin{tabular}{cc}
\hline$\beta_{0}$ & $\beta_{1}$ \\
\hline 1.005 & 0.377 \\
$(1.304)^{*}$ & $(4.226)$ \\
\hline
\end{tabular}

* Los números entre paréntesis son los estadísticos t. Estimaciones realizadas en J-Multi 4.23.

A decir de los coeficientes del vector cointegrante, el índice de precios y cotizaciones (IPC) impacta de manera positiva en la actividad económica, medida a partir del índice de volumen de la producción industrial (IVPI), en una proporción menor a la unidad, mientras los coeficientes alpha, que miden la velocidad de ajuste del término de corrección de error en el MCE y permiten realizar pruebas de exogeneidad débil, se muestran en el cuadro 5:

Cuadro 5. Coeficientes alpha del MCE

\begin{tabular}{cc}
\hline$i v p i$ & $i p c$ \\
\hline 0.002 & 0.041 \\
$(0.680)^{*}$ & $(4.931)$ \\
\hline
\end{tabular}

* Los números entre paréntesis son los estadísticos $t$. 
Como se puede apreciar en el cuadro 5, el único coeficiente de velocidad de ajuste estadísticamente significativo es el correspondiente a ipc, de esta manera ivpi resultó ser exógena débil, lo cual corrobora la dirección de la causalidad especificada en (1).

Los cuadros 6 y 7 muestran los resultados de la prueba de normalidad y el cuadro 8 presenta los resultados de las pruebas de autocorrelación y de heterocedasticidad del modelo. Como se puede apreciar en dichos cuadros, los residuos cumplen con los supuestos de normalidad; de igual forma, las pruebas LM realizadas tampoco revelan autocorrelación al modelo hasta con 12 rezagos con el fin de verificar que no tengan alguna posible autocorrelación de tipo estacional ya que se trata de datos mensuales, sin embargo, el modelo no superó las pruebas de heterocedasticidad condicional autorregresiva en forma conjunta. ${ }^{2}$ El hecho de que el modelo cumpla en su mayoría con estos supuestos permite realizar inferencia económica válida en el MCE.

Cuadro 6. Pruebas de normalidad para los residuos del MCE*

\begin{tabular}{ccccc}
\hline & \multicolumn{2}{c}{ Doornik y Hansen (1994) } & \multicolumn{2}{c}{ Lütkepohl (1993) } \\
\hline Prueba & Estadístico & Probabilidad & Estadístico & Probabilidad \\
Conjunta & 7.5316 & {$[0.1103]$} & 4.7197 & {$[0.3173]$} \\
Asimetría & 3.9235 & {$[0.1406]$} & 2.3991 & {$[0.3013]$} \\
Curtosis & 3.6081 & {$[0.1646]$} & 2.3206 & {$[0.3134]$} \\
\hline
\end{tabular}

* Pruebas realizadas en J-Multi 4.23.

Cuadro 7. Pruebas de normalidad Jarque-Bera para los residuos del MCE*

\begin{tabular}{ccccc}
\hline Ecuación & Estadístico-t & Valor- $p$ de $\chi^{2}$ & Asimetría & Curtosis \\
\hline$\Delta i n p i_{t}$ & 2.3966 & {$[0.3017]$} & 0.0468 & 3.4847 \\
$\Delta i p c_{t}$ & 4.3354 & {$[0.1144]$} & -0.3175 & 3.1943 \\
\hline
\end{tabular}

* Pruebas realizadas en J-Multi 4.23.

\footnotetext{
${ }^{2}$ Este tipo de heterocedasticidad es característico de las series de tiempo financieras, por lo que no es de extrañar dicho resultado.
} 
Cuadro 8. Pruebas de diagnóstico del MCE de manera conjunta a

\begin{tabular}{ccc}
\hline Prueba & Estadístico & Probabilidad \\
\hline Autocorrelación & $L M$ & \\
LM (1) & 1.7979 & {$[0.7729]$} \\
LM (2) & 7.3720 & {$[0.4971]$} \\
LM (3) & 15.0561 & {$[0.2384]$} \\
LM (4) & 18.8953 & {$[0.2741]$} \\
LM (5) & 19.8355 & {$[0.4683]$} \\
LM (6) & 32.3375 & {$[0.1188]$} \\
LM (7) & 36.3516 & {$[0.1339]$} \\
LM (8) & 36.9086 & {$[0.2523]$} \\
LM (9) & 40.5228 & {$[0.2776]$} \\
LM (10) & 49.9306 & {$[0.1350]$} \\
LM (11) & 51.9357 & {$[0.1922]$} \\
LM (12) & 57.6145 & {$[0.1612]$} \\
Heterocedasticidad & & \\
VARCH - LM (1) & 24.5729 & {$[0.0035]$} \\
VARCH - LM (2) & 33.9120 & {$[0.0129]$} \\
VARCH - LM (3) & 41.0274 & {$[0.0409]$} \\
VARCH - LM (4) & 47.2821 & {$[0.0988]$} \\
\hline
\end{tabular}

${ }^{a}$ Los números entre paréntesis son los rezagos incorporados en cada prueba.

Cuadro 9. Pruebas univariadas $A R C H-L M^{a}$

\begin{tabular}{lcccc}
\hline & Estadistico-t & Probabilidad & Estadistico F & Probabilidad \\
\hline$\hat{u}_{1 t}$ & 7.4734 & ARCH-LM (1) & \\
$\hat{u}_{2 t}$ & 2.4017 & {$[0.0063]$} & 7.7189 & {$[0.0059]$} \\
& \multicolumn{5}{c}{ ARCH-LM (2) } & 2.4265 & {$[0.1207]$} \\
$\hat{u}_{1 t}$ & 9.1424 & {$[0.0103]$} & 4.7570 & {$[0.0095]$} \\
$\hat{u}_{2 t}$ & 2.7243 & {$[0.2561]$} & 1.3782 & {$[0.2541]$} \\
& & $A R C H-L M(3)$ & & \\
$\hat{u}_{1 t}$ & 10.3941 & {$[0.0162]$} & 3.5936 & {$[0.0144]$} \\
$\hat{u}_{2 t}$ & 2.6474 & {$[0.4492]$} & 0.8926 & {$[0.4457]$} \\
\hline
\end{tabular}

a Pruebas realizadas en J-Multi 4.23 . 
El cuadro 9 presenta los resultados de las pruebas univariadas ARCH-LM, de las cuales se puede deducir que la presencia de heterocedasticidad en el MCE estimado se atribuye a que los residuos de la ecuación del índice del volumen de la producción industrial presentan efectos de heterocedasticidad condicional autorregresiva, conocidos comúnmente como efectos ARCH.

Por último, el cuadro 10 muestra los resultados de la prueba de no causalidad en el sentido de Granger en el MCE. Como se puede ver, la prueba no permite rechazar la hipótesis nula de que el ipc no causa en el sentido de Granger al ivpi.

Cuadro 10. Pruebas de no causalidad en el sentido de Granger

\begin{tabular}{ccc}
\hline Hipótesis nula: & $\chi^{2}(5)=$ & Valor-p \\
\hline ipc no causa a ivpi & $1.7889 *$ & 0.0889 \\
ivpi no causa a ipc & 0.5803 & 0.7721 \\
\hline
\end{tabular}

Los asteriscos * $y * *$ indican el rechazo de la hipótesis nula al 5 y al $1 \%$ de significación, respectivamente.

Este resultado implica que el modelo no provee fuerte evidencia sobre la hipótesis de que la causalidad vaya del ipc al ivpi. No obstante, como se mencionó anteriormente, el coeficiente de velocidad de ajuste del MCE sí reacciona al término de corrección del error.

Dado que los residuos del MCE presentaron efectos ARCH, tanto de manera individual como en forma conjunta, y procediendo de manera análoga a como se realiza cuando un modelo uniecuacional presenta este tipo de heterocedasticidad condicional autorregresiva, optamos por modelar las relaciones entre el indicador bursátil y el índice del volumen de producción industrial tratando de capturar dichos efectos a partir de un GARCH multivariado (MGARCH por Multivariate GARCH), el cual se puede escribir de la siguiente forma:

$$
\sum_{t}=\Gamma_{0}^{\prime} \Gamma_{0}+\sum_{n=1}^{N} \sum_{i=1}^{q} \Gamma_{n i}^{\prime} u_{t-i} u_{t-i}^{\prime} \Gamma_{n i}+\sum_{n=1}^{N} \sum_{i=1}^{q} B_{n i}^{\prime} \sum_{t-i} B_{n i} .
$$

Las estimaciones del modelo MGARCH para los residuos del MCE se presentan en el cuadro 11. 
Cuadro 11. Estimación del MGARCH para los residuos del MCE a

\begin{tabular}{rrrrrrr}
\hline & \multicolumn{2}{c}{ Parámetros } & \multicolumn{2}{c}{ MLt-ratios } & \multicolumn{2}{c}{ QML t-ratios } \\
\hline$\hat{\Gamma}_{0}$ & 0.0063 & 0.0046 & -1.6116 & -0.1688 & -2.2751 & -1.6978 \\
& --- & 0.0013 & & -0.0088 & & -5.0527 \\
$\hat{\Gamma}_{1}$ & 0.1723 & 0.0590 & -1.5527 & -0.1855 & -1.2510 & -0.4225 \\
& 0.0638 & 0.1392 & -1.8667 & -1.4680 & -2.5562 & -2.6265 \\
$\hat{B}_{1}$ & 0.9234 & 0.3279 & -16.4766 & -1.9443 & -17.4898 & -3.9877 \\
& -0.0554 & 0.9701 & -2.2199 & -22.5213 & -3.8064 & -72.8043 \\
\hline
\end{tabular}

${ }^{\text {a }}$ Estimaciones realizadas en J-Multi 4.23.

Los resultados del modelo MGARCH, de acuerdo con Lütkepohl y Krätzig (2004), se interpretan del siguiente modo: la dinámica de sección cruzada se puede obtener de los parámetros que se encuentran fuera de la diagonal principal de las matrices $\hat{\Gamma}_{1}$ y $\hat{B}_{1}$; si estos parámetros son estadísticamente significativos, entonces los errores de cada una de las variables, en este caso del MCE, impactan a la otra variable. De esta manera, la ecuación de la varianza del índice de volumen de la producción industrial se ve afectada por los residuos de la ecuación correspondiente al índice de precios y cotizaciones del mercado bursátil mexicano ya que su parámetro, de 0.0638 , resultó estadísticamente significativo, con un estadístico $t$ de -2.56 , de acuerdo con los estadísticos $t$ del método de cuasi máxima verosimilitud. De igual forma, del modelo estimado se puede inferir que hay una transmisión simultánea de volatilidades entre ambas variables, como lo sugiere la significancia estadística de los parámetros que se encuentran fuera de la diagonal principal correspondientes a la matriz $\hat{B}_{1}$. No obstante, dado que los residuos del MCE resultaron distribuirse en forma normal, la evidencia obtenida a partir de los estadísticos $t$ de máxima verosimilitud puede considerarse suficiente; siendo éste el caso, la transmisión de la volatilidad va únicamente del mercado de capitales a la actividad económica y no a la inversa.

\section{CONCLUSIONES}

La investigación sobre el desarrollo financiero y crecimiento económico es abundante pero no concluyente para determinar si el sistema financiero es un motor 
que impulsa el crecimiento económico, si es a la inversa, o bien si se determinan de manera simultánea. Dentro de esta discusión, se encuentra presente la cuestión de si el desarrollo del mercado de valores, como parte del sistema financiero, promueve el crecimiento económico, si ocurre lo contrario o bien si ambas variables se realimentan.

Para responder la anterior cuestión en el caso mexicano empleamos herramientas de la econometría dinámica moderna, la cual provee un marco bien definido para determinar la posible dirección de causalidad entre un conjunto de variables.

Nuestros resultados son consistentes con lo encontrado por Ron (2001), en el sentido de que el IPC causa al IVPI, ya que es posible rechazar la hipótesis nula de exogeneidad débil para esta variable, pero a diferencia de este último autor nosotros sólo encontramos evidencia de causalidad en el sentido de Granger al nivel de significación de $10 \%$, de acuerdo con los resultados del modelo de corrección del error.

En tanto que los residuos del MCE estimado presentaron efectos ARCH, estimamos un modelo GARCH multivariado (MGARCH) para estos residuos. Al realizar la estimación del MGARCH encontramos evidencia adicional de que la volatilidad del IPC incide en la volatilidad del IVPI y que la transmisión de las volatilidades entre ambas variables se da en ambos sentidos.

De esta manera, la evidencia encontrada en nuestro estudio sugiere que el desarrollo del mercado de valores incide de manera positiva y promueve la actividad económica industrial, aunque nuestros resultados no son del todo concluyentes ya que tal hipótesis no se pudo rechazar en los niveles convencionales de significación, por lo que se requiere mayor investigación haciendo uso de otros indicadores que puedan dar cuenta del desarrollo del mercado de valores. 


\section{REFERENCIAS BIBLIOGRÁFICAS}

Aylward, A., y J. Glen (2000), "Some International Evidence on Stock Prices as Leading Indicators of Economic Activity", Applied Financial Economics, núm. 10, pp. 1-14.

Bencivenga, V. R., y B. D. Smith (1991), "Financial Intermediation and Endogenous Growth", The Review of Economic Studies, núm. 58, pp. 195-209.

Binswanger, M. (2000), “Stock Returns and Real Activity: Is There Still a Connection?", Applied Financial Economics, núm. 10, pp. 379-387.

Buffie, E. F. (1984), "Financial Repression, the New Structuralists, and Stabilization Policy in Semi-industrialized Economics", Journal of Development Economics, núm. 14, pp. 305-322.

Caporale, Howells y Soliman (2004), "Stock Market Development and Economic Growth: A Casual Linkage", Journal of Economic Development, núm. 29, pp. 33-50.

Chen, N. F., R. Roll, y S. A. Ross (1986), "Economic Forces and the Stock Market", Journal of Business, núm. 59, pp. 383-403.

Choi, J., S. Hauser, y K. J. Kopecky (1999), "Does the Stock Market Predict Real Activity? Time Series Evidence from the G-7 Countries", Journal of Banking and Finance, núm. 23, pp. 1771-1792.

Chumacero, Rómulo (2000), "Se busca una raíz unitaria: Evidencia para Chile”, Estudios de Economía, Vol. 27, núm. 001, junio, Universidad de Chile, Santiago, pp. 55-68.

Cuthbertson, K., S. G. Hall, y M. P. Taylor (1992), Applied Econometric Techniques, Nueva York, Simon and Schuster.

Dickey, D. A., y W. A. Fuller (1981), "Likelihood Ratio Statistics for Autoregressive Time Series With a Unit Root”, Econometrica, núm. 49, pp. 1057-1072.

Doornik, J. A., y H. Hansen (1994), “A Practical Test of Multivariate Normality", Nuffield College, documento inédito.

Enders, Walter (1995), Applied Econometric Time Series, John Wiley and Sons, Inc.

Fama, Eugene F. (1981), "Stock Returns, Real Activity, Inflation and Money", The American Economic Review, Vol. 71, núm. 4, pp. 545-565.

- (1990), "Stock Returns, Expected Returns, and Real Activity", Journal of Finance, núm. 45, pp. 1089-1108.

Goldsmith, R. (1969), Financial Structure and Development, New Haven, Yale University Press.

Guha Deb, Soumya, y J. Mukherjee (2008), “Does Stock Market Development Cause Economic Growth? A Time Series Analysis for Indian Economy”, International Research Journal of Finance and Economics, núm. 21, pp. 142-149. 
Habibullah, M. S., y A. Z. Baharumshah (1996), "Money, Ouput and Stock Prices in Malasia: An Application of the Cointegration Test", International Economic Journal, núm. 10 (2), pp. 121-130.

Hondroyannis, George, y Evangelina Papapetrou (2001), "Macroeconomic Influences in the Stock Market”, Journal of Economics and Finance, núm. 25 (1), pp. 33-49.

James, C., S. Koreisha, y M. Partch (1985), "A VARMA analysis of the causal relations among stock returns, real output, and nominal interest rates", Journal of Finance, núm. 40, pp. 1375-1384

Johansen, Soeren (1988), "Statistical and Hypothesis Testing of Cointegration Vectors", Journal of Economic Dynamics and Control, núm. 12, pp. 231-254.

(1992 a), "Cointegration in Partial Systems and the Efficiency of Single Equation Analysis", Journal of Econometric, núm. 52, pp. 389-402.

(1992b), "Testing Structural Hypotheses in a Multivariate Cointegration Analysis at the Purchasing Power Parity and the Uncovered Interest Parity for the UK", Journal of Econometrics, núm. 53, pp. 211-244.

— y K. Juselius (1990), "Maximum Likelihood Estimation and Inference on Cointegration - with Applications to the Demand for Money", Oxford Bulletin of Economics and Statistics, núm. 52, pp. 169-210.

King, R., y R. Levine (1993 a), "Finance and Growth: Schumpeter Might Be Right", Quarterly Journal of Economics, núm. 108 (3), pp. 717-738.

- (1993b), "Finance, Entrepreneurship and Growth Theory and Evidence", Journal of Monetary Economics, núm. 32 (3), pp. 513-542.

Kwiatkowsky, D., P. C. B. Phillips, P. Schmidt, y Y. Shin (1992), "Testing the null hypothesis of stationary against the alternative of a unit root", Journal of Econometrics, Vol. 1, pp. 159-178.

Lee, B. S. (1992), “Causal Relations among Stock Returns, Interest Rates, Real Activity and Inflation”, Journal of Finance, núm. 47, pp. 1591-1603.

Levine, Ross (1997), "Financial Development and Economic Growth: Views and Agenda", Journal of Economic Literature, núm. 35, pp. 688-726.

y Sarah Zervos (1998), "Stock Markets, Banks, and Economic Growth", American Economic Review, núm. 88, pp. 537-558.

Lucas, R. E. (1988), “On the Mechanics of Economic Development”, Journal of Monetary Economics, 22, pp. 3-42.

Lütkepohl, Helmut (1993), Introduction to Multiple Time Series Analysis, Nueva York, Londres y Tokio, Heidelberg, primavera, segunda edición.

y Marcus Krätzig (2004), Applied Time Series Econometrics, Cambridge, Cambridge University Press. 
McKinnon, R. I. (1973), Money and Capital in Economic Development, Washington, The Brookings Institution.

Méndez Montaño, Saúl (2007), "El mercado bursátil y su relación con el crecimiento económico de México, 1995-2005”, Universidad Nacional Autónoma de México, Facultad de Contaduría y Administración, tesis de maestría.

Mirakhor, S., y R. M. Villanueva (1990), "Market Integration and Investment Barriers in Emerging Equity Markets", World Bank Discussion Paper, núm. 216, pp. 221255.

Mishkin, S. Frederic (2001), The Economics of Money, Banking, and Financial Markets, Nueva York, Addison Wesley Longman, 6a. ed.

Mosconi R., S. Johansen, y B. Nielsen (2000), "Cointegration Analysis in the Presence of Structural Breaks in the Deterministic Trend", Econometrics Journal, núm. 3, Vol. 2, pp. 216-249.

Odedokun, M. O. (1996), “Alternative Econometric Approaches for Analyzing the Role of the Financial Sector in Economic Growth: Time-Series Evidence from LDCs", Journal of Development Economics, núm. 50 (1), pp. 119-135.

Osei, Victor (2004), Does the Stock Market Matter in Ghana? A Granger-Causality Analysis, Bank of Ghana, WP/BOG-05/13.

Pardy, R. (1992), "Institutional Reform in Emerging Securities Markets", Policy Research Working Papers (Financial Policy and Systems), núm. 907, Banco Mundial.

Rajan, R. G., y L. Zingales (1998), "Financial Dependence and Growth", American Economic Review, núm. 88, pp. 559-586.

Robinson, J. (1952), "The Generalization of the General Theory”, The Rate of Interest and Other Essays, Londres, Macmillan, pp. 69-142.

Rodríguez Benavides, Domingo, y Francisco López Herrera (2009), "Desarrollo financiero y crecimiento económico en México", Problemas del Desarrollo, Vol. 40, núm. 159, pp. 39-60.

Ron, Francisco, E. (2001), “Ajuste dinámico y equilibrio entre la producción industrial y la actividad bursátil en México", Momento Económico, núm. 118, noviembrediciembre, pp. 21-38.

Schumpeter, J. A. (1911), The Theory of Economic Development, Cambridge, Mass., Harvard University Press.

Schwert, G. W. (1990), "Stock Returns and Real Activity: A Century of Evidence", Journal of Finance, núm. 45, pp. 1237-1254.

Shahbaz, Muhammad, N. Ahmed, y L. Ali (2008), "Stock Market Development and Economic Growth: Ardl Causality in Pakistan”, International Research Journal of Finance and Economics, núm. 14, pp. 182-195. 
54 ECONOMÍA: TEORÍA Y PRÁCTICA • Nueva Época, número 32, enero-junio 2010

Shaw, E. S. (1973), Financial Deepening in Economic Development, Nueva York, Oxford University Press.

Singh, A. (1997), "Financial Liberalization, Stock Markets and Economic Development", The Economic Journal, núm. 107, pp. 771-782.

Stern, N. (1989), “The economics of development: a survey”, Economic Journal, núm. 100, pp. 597-685.

Thornton, J. (1993), "Money, Output and Stock Prices in the UK: Evidence on Some (Non) Relationships", Applied Financial Economics, núm. 3, pp. 335-338.

Toda, H. Y., y Yamamoto (1995), "Statistical Inference in Vector Autoregressions with Possibly Integrated Processes", Journal of Econometrics, número 66, páginas 225-250.

Van Wijnbergen, S. (1983), "Credit Policy, Inflation and Growth in Financially Repressed Economy", Journal of Development Economics, núm. 13, pp. 45-65.

Xu, Z. (2000), "Financial Development, Investment, and Economic Growth", Economic Inquiry, núm. 38 (2), p. 332. 